\title{
Etiology and Antibiotic Susceptibility Patterns of Community- and Hospital-Acquired Urinary Tract Infections in a General Hospital in Kuwait
}

\author{
Khalifa Al Benwan ${ }^{\text {a Noura Al Sweih }}{ }^{\text {b }}$ Vincent O. Rotimi ${ }^{\text {b }}$ \\ ${ }^{a}$ Department of Microbiology, Al-Amiri Hospital, and ${ }^{\text {b}}$ Faculty of Medicine, Kuwait University, Kuwait
}

\author{
Key Words \\ Etiology · Antibiotic susceptibility • Urinary tract infection • \\ Community $\cdot$ Hospital, Kuwait
}

\begin{abstract}
Objective: Our purpose was to determine the bacterial profile and prevalence of antibiotic resistance patterns of uropathogens, as well as evaluate the problem with extendedspectrum $\beta$-lactamase (ESBL) producing isolates, causing urinary tract infections (UTIs) in Al-Amiri Hospital, Kuwait, over a 3-year period. Materials and Methods: Isolates $(56,505)$ from symptomatic UTI cases from January 2005 to December 2007 were identified by conventional methods and the VITEK identification card system. Antimicrobial susceptibility testing was performed by disk diffusion method for Gram-positive organisms and an automated VITEK 2 machine for Gram-negative organisms. ESBL production by the Enterobacteriaceae was detected by the double-disk diffusion method and VITEK-2 system. Results: Significant bacteriuria was detected in 15,064 (26.6\%) of the 56,505 urine samples. Escherichia coli accounted for 4,876 (54.9\%) from community-acquired UTI (CA-UTI) and 2,253 (36.4\%) from hospital-acquired UTI (HA-UTI), followed by Streptococcus agalactiae (1,129, 12.7\%) and Klebsiella pneumoniae (962, $10.8 \%)$ from CA-UTI cases. Candida spp. $(973,15.7 \%)$ and $K$. pneumoniae (747, 12.1\%) were the second and third most prevalent isolates, respectively, in HA-UTI. High resistance rates were observed among the Enterobacteriaceae against ampicillin, cephalothin, ciprofloxacin, piperacillin and tri-
\end{abstract}

methoprim-sulfamethoxazole. About 855 (12\%) and 291 (17\%) of E. coli and K. pneumoniae, respectively, were resistant to $\geq 4$ antibiotics. The prevalence of ESBL-producing E. coli and K. pneumoniae in CA-UTI was 12 and $17 \%$ and in HA-UTI 26 and 28\%, respectively. Conclusion: A high percentage of the uropathogens causing UTI in the Al-Amiri Hospital setting was highly resistant to the first- and secondline antibiotics for the therapy of UTI. ESBL-producing bacteria are highly prevalent in our hospital.

Copyright $\odot 2010$ S. Karger AG, Basel

\section{Introduction}

Urinary tract infections (UTIs) are one of the common bacterial infections encountered by both general practitioners and hospital doctors [1]. Acute UTIs are associated with substantial morbidity and problems of recurrent infections. Studies have shown that approximately $25 \%$ of the women with the first UTI will have another attack within 6 months [2]. In addition, UTIs are an important cause of septicemia resulting in high mortality rates, prolonged hospital stays and increased healthcare costs [2]. In almost all cases, treatment must be initiated before the final bacteriological results are available particularly in the outpatient setting. To optimize the use of empiric antibacterial therapy for UTI, current knowledge of the organisms that cause UTI and their antibiotic susceptibility is essential. This is mainly because the etiology of UTI and the antibiotic susceptibility of the causative

\section{KARGER}

Fax +4161306 1234

E-Mail karger@karger.ch

www.karger.com (c) 2010 S. Karger AG, Basel

$1011-7571 / 10 / 0196-0440 \$ 26.00 / 0$

Accessible online at:

www.karger.com/mpp
Dr. Khalifa Al Benwan, MD, MSc, FRCPath

Department of Microbiology

Al-Amiri Hospital, PO Box 4077

Safat 13041 (Kuwait)

Tel. +965 6691 0508, Fax +965 2246 3790, E-Mail drbanwan@gmail.com 
Table 1. Gender and age distribution of patients with UTIs in a general hospital in Kuwait (percentages in parentheses)

\begin{tabular}{|c|c|c|c|c|c|c|c|c|}
\hline \multirow[t]{2}{*}{ Gender } & \multicolumn{7}{|l|}{ Age, years } & \multirow[t]{2}{*}{ Total } \\
\hline & $0-9$ & $10-19$ & $20-29$ & $30-39$ & $40-49$ & $50-59$ & $\geq 60$ & \\
\hline Male & $308(21)$ & $103(17)$ & $231(11)$ & $360(18)$ & $360(28)$ & $771(35)$ & $1,851(35)$ & $3,984(27)$ \\
\hline Total & $1,465(10)$ & $617(4)$ & 2,159 (14) & $2,031(13)$ & $1,311(9)$ & $2,237(15)$ & $5,244(35)$ & $15,064(100)$ \\
\hline
\end{tabular}

uropathogens have been changing over the past years, both in community and nosocomial infections [3]. However, the information on the etiology and resistance pattern of UTI isolates in Kuwait is not current.

The aim of the study was to determine the local bacterial species distribution of UTI isolates and their susceptibility pattern to antibiotics.

\section{Material and Methods}

The study was performed in the Department of Microbiology of Al-Amiri Hospital, a 400-bedded teaching hospital, including a 15-bedded general intensive care unit, urology unit, renal dialysis and kidney transplant units. Al-Amiri Hospital serves a population of approximately 400,000 people of different nationalities and provides clinical laboratory services to 22 polyclinics. To reduce the bias due to different pathogen distributions and the susceptibility profile associated with in-/outpatient status, patients were assigned to 2 categories: CA-UTI and HA-UTI. The samples $(56,505)$ were collected between January 2005 and December 2007 from both the inpatients and the outpatients. In the laboratory, all samples were inoculated onto a set of CLED (Oxoid, Basingstoke, UK) and blood agar (Oxoid) plates and incubated in air at $37^{\circ} \mathrm{C}$ for $24 \mathrm{~h}$. Urine specimens with bacterial growth of $\geq 10^{4}$ $\mathrm{cfu} / \mathrm{ml}$ were regarded as significant bacteriuria [4].

All Gram-negative bacteria were identified and antibiotic susceptibility testing was performed by an automated VITEK-2 machine (bioMérieux, Marcy-I’Étoile, France); A VITEK 2 GN card was used for identification and an AST-N020 card for susceptibility testing (bioMérieux). All Gram-positive bacteria were identified by standard biochemical tests and sensitivity of the organisms determined by Kirby-Bauer method. The results were interpreted according to the recommendation of the Clinical and Laboratory Standards Institute [5]. Extended-spectrum $\beta$-lactamase (ESBL) activity was detected by the double-desk synergy method using amoxicillin/clavulanic acid and cefotaxime and ceftazidime disks. The ESBL-producing strains were confirmed with the automated VITEK-2 system according to the manufacturer's instructions. For the quality control of susceptibility tests E. coli ATCC 25922, Pseudomonas aeruginosa ATCC 27853, Staphylococcus aureus ATCC 25923 and Enterococcus faecalis ATCC929212 strains were used. Discrete variables were expressed as percentages and the $\chi^{2}$ test was used to compare proportions.

In vitro Activity of Several Antibiotics against Uropathogens in Amiri Hospital

\section{Results}

Of the 56,505 urine samples processed during the study period, 15,064 (26.6\%) yielded significant bacteriuria. Of the 15,064 samples, 6,180 (41.0\%) were from inpatients (HA-UTI) and 8,884 (59.0\%) were from outpatients (CA-UTI). Most cases of UTIs were recorded among young and middle age patients (20-59 years, $51 \%)$. Pediatric patients ( $0-19$ years) comprised 2,082 (14\%) and elderly ( $\geq 60$ ) constituted 5,244 (35\%) of the total number of UTIs. A significantly higher incidence of UTI was observed in females compared with males, 11,080 (73\%) and 3,984 (27\%), respectively (table 1 ).

\section{Etiological Isolates}

Gram-negative aerobic rods accounted for 10,726 (71.2\%) of the total pathogens, Gram-positive cocci for 3,179 (21.1\%) and Candida spp. for 1,160 (7.7\%). The detailed analysis of the etiological agents is shown in table 2 . E. coli was the predominant pathogen in both CA-UTI and HA-UTI cases, accounting for 4,876 (54.9\%) and $2,253(36.4 \%)$ of the causative agents, respectively. The next top 5 uropathogens, after E. coli, causing HA-UTI in our hospital were Candida spp.: 973 (15.7\%); K. pneumoniae: 747 (12.0\%); Enterococcus spp.: 713 (11.5\%); P. aeruginosa: 609 (9.8\%) and S. agalactiae: 326 (5.2\%), while those causing CA-UTI were $S$. agalactiae $(1,129,12.7 \%)$; K. pneumoniae (962, 10.8\%); Enterococcus spp. (709, 7.9\%); P. aeruginosa $(386,4.3 \%)$ and Candida spp. (178, $2.3 \%)$

\section{Antimicrobial Susceptibility Pattern}

The susceptibility of the Gram-negative bacterial isolates, expressed as resistance rates, is shown in table 3. Generally, Gram-negative bacteria from inpatients were more resistant to antimicrobials than those from outpatients.

Enterobacteriaceae. The E. coli resistance rates against the so-called first-line antibiotics were: amoxicillin (66 
Table 2. Microbial uropathogens isolated from urines of inpatients and outpatients with urinary tract infection (UTI)

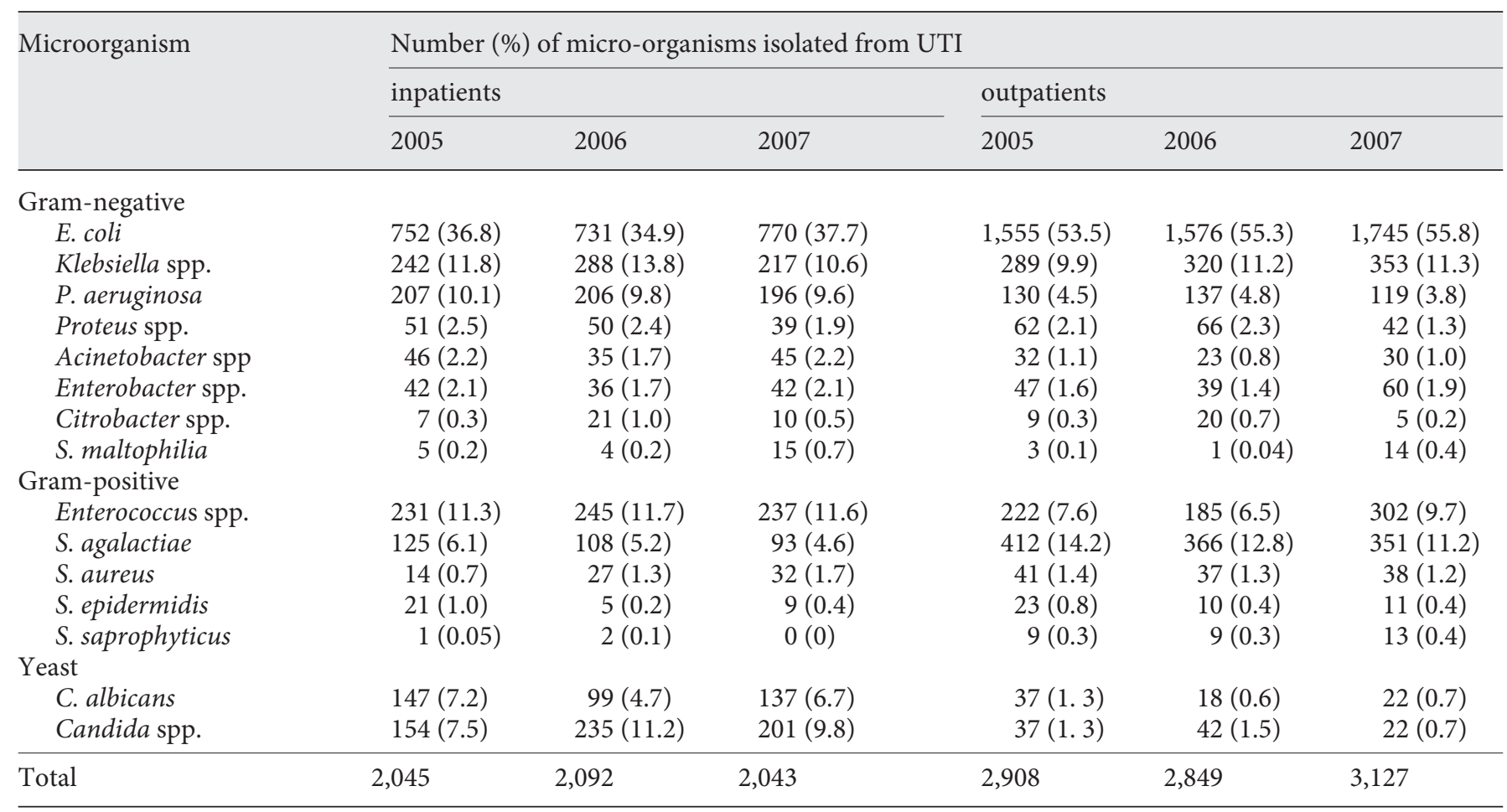

vs. 75\%), trimethoprim-sulfamethoxazole (TMP-SMX) (47 vs. $52 \%$ ), ciprofloxacin ( 28 vs. $46 \%$ ), cephalothin (47 vs. $60 \%$ ), amoxicillin-clavulanic acid (27 vs. $41 \%$ ) and cefotaxime (12 vs. $26 \%$ ). Amikacin (1 vs. $3 \%$ ), piperacillintazobactam (1 vs. $4 \%$ ) and nitrofurantoin (7 vs. $8 \%$ ) had excellent activities against $E$. coli. Overall, K. pneumoniae causing CA-UTI as well as HA-UTI had high resistance rates against TMP-SMX (26 vs. $29 \%$ ), ciprofloxacin (23 vs. $37 \%$ ), cephalothin ( 27 vs. $41 \%$ ), amoxicillin-clavulanate ( 21 vs. $33 \%)$ and cefotaxime (17 vs. $28 \%)$. The resistance rates of $K$. pneumoniae to piperacillin-tazobactam were 6 vs. $16 \%$ and to amikacin 2 vs. $6 \%$.

Nonfermenting Gram-Negative Bacterial Isolates. As for the P. aeruginosa isolates from both CA-UTI and HAUTI, the resistance rates to amikacin were 10 and $15 \%$, to piperacillin-tazobactam 10 and $14 \%$, and to ciprofloxacin 30 and $28 \%$, respectively. Acinetobacter isolates from CAUTI were less resistant than those from HA-UTI to several antibiotics. For example, compared to isolates from CA-UTI, the resistance rates of those from HA-UTI were 7 vs. $28 \%$ against amikacin, 10 vs. $42 \%$ to piperacillintazobactam, 12 vs. $47 \%$ to ciprofloxacin and 7 vs. $33 \%$ to TMP-SMX.
Gram-Positive Bacterial Isolates. The resistance rates of Gram-positive bacterial isolates are shown in table 4 . All the Gram-positive isolates were susceptible to vancomycin. None of the S. agalactiae isolates was resistant to penicillin. Although all the Enterococcus spp. were susceptible to vancomycin, teicoplanin and nitrofurantoin, the resistance rates to penicillin by isolates from CA-UTI and HA-UTI were 24 and 35\%, respectively. The majority of both CA-UTI and HA-UTI isolates of S. aureus exhibited high susceptibility rates; the resistance rates of cephalexin were 0 and $2 \%$, to cloxacillin 0 and $3 \%$ and to TMPSMX 3 and $2 \%$.

\section{Prevalence of ESBL-Producing Isolates}

The number of the members of the family of Enterobacteriaceae that were positive for ESBL production by the double disc synergy (DDS) test essentially tallied with those detected by the VITEK-2 system. Approximately 585 (12\%) of the E. coli isolated from CA-UTI and 586 (26\%) from HA-UTI were ESBL producers. A relatively high proportion of $K$. pneumoniae from CA-UTI 164 (17\%) and HA-UTI 209 (28\%) were also ESBL producers. The resistance of ESBL-producing E. coli and K. pneumoniae is shown in table 5 . 
Table 3. Frequency and percentage of resistant Gram-negative uropathogens isolated from outpatients vs. inpatients with UTIs in Al-Amiri Hospital

\begin{tabular}{|c|c|c|c|c|c|c|c|c|c|c|c|c|c|c|c|}
\hline Bacteria & Year & $\mathrm{CA} / \mathrm{HA}$ & \multicolumn{13}{|c|}{ Percentage of CA vs. HA bacteria resistant to } \\
\hline \multirow[t]{3}{*}{ E.coli } & 2005 & $1,555 / 752$ & $1 / 1$ & $27 / 43$ & $66 / 72$ & $47 / 60$ & $9 / 30$ & $27 / 33$ & $26 / 43$ & $47 / 47$ & $12 / 23$ & $10 / 18$ & $5 / 7$ & $43 / 57$ & $1 / 5$ \\
\hline & 2007 & $1,745 / 770$ & $1 / 3$ & $28 / 44$ & $68 / 78$ & $50 / 62$ & $17 / 27$ & & $30 / 50$ & $47 / 53$ & $15 / 26$ & $44 / 62$ & $9 / 8$ & $48 / 59$ & $1 / 4$ \\
\hline & & $4,876 / 2,253$ & $1 / 3$ & $27 / 41$ & $66 / 75$ & $47 / 60$ & $12 / 26$ & $9 / 11$ & $28 / 46$ & $47 / 52$ & $14 / 25$ & $22 / 33$ & $7 / 8$ & $50 / 62$ & $1 / 4$ \\
\hline \multirow{2}{*}{ K. pneumoniae } & 2007 & $353 / 217$ & $1 / 7$ & $24 / 29$ & $100 / 100$ & $31 / 34$ & $20 / 33$ & & $26 / 43$ & $26 / 32$ & $14 / 13$ & $28 / 33$ & $65 / 66$ & $88 / 84$ & $6 / 14$ \\
\hline & & $962 / 747$ & $2 / 6$ & $21 / 33$ & $100 / 100$ & $27 / 41$ & $17 / 28$ & $4 / 12$ & $23 / 37$ & $26 / 29$ & $12 / 22$ & $15 / 20$ & $56 / 64$ & $85 / 86$ & $6 / 16$ \\
\hline \multirow[t]{4}{*}{ P. aeruginosa } & 2005 & $130 / 207$ & $10 / 21$ & - & - & - & - & - & $33 / 30$ & - & $23 / 27$ & - & - & $16 / 24$ & $9 / 16$ \\
\hline & 2006 & $137 / 206$ & $7 / 9$ & - & - & - & - & - & $22 / 25$ & - & $15 / 17$ & - & - & $13 / 19$ & $7 / 11$ \\
\hline & 2007 & $119 / 196$ & $13 / 14$ & - & - & - & - & - & $35 / 30$ & - & $31 / 21$ & - & - & $17 / 24$ & $13 / 16$ \\
\hline & & $386 / 609$ & $10 / 15$ & - & - & - & - & - & $30 / 28$ & - & $23 / 22$ & - & - & $15 / 22$ & $10 / 14$ \\
\hline \multirow[t]{4}{*}{ P. mirabilis } & 2005 & $62 / 51$ & $0 / 0$ & $0 / 0$ & $35 / 35$ & $11 / 10$ & $0 / 0$ & - & $31 / 25$ & $48 / 35$ & $16 / 16$ & $2 / 9$ & $98 / 100$ & $10 / 4$ & $0 / 0$ \\
\hline & 2006 & $66 / 50$ & $0 / 4$ & $5 / 16$ & $23 / 54$ & $12 / 28$ & $3 / 14$ & - & $18 / 42$ & $32 / 52$ & $14 / 26$ & $9 / 21$ & $97 / 100$ & $18 / 32$ & $2 / 0$ \\
\hline & 2007 & $42 / 39$ & $3 / 0$ & $3 / 6$ & $34 / 25$ & $8 / 6$ & $3 / 8$ & - & $11 / 28$ & $32 / 36$ & $11 / 14$ & $21 / 34$ & $67 / 100$ & $8 / 8$ & $0 / 0$ \\
\hline & & $170 / 140$ & $1 / 1$ & $3 / 7$ & $30 / 39$ & $11 / 15$ & $2 / 7$ & - & $21 / 32$ & $38 / 41$ & $14 / 19$ & $9 / 20$ & $90 / 100$ & $13 / 15$ & $1 / 0$ \\
\hline \multirow[t]{4}{*}{ Enterobacter spp. } & 2005 & $47 / 42$ & $0 / 0$ & $100 / 98$ & $100 / 100$ & $98 / 98$ & $22 / 45$ & - & $13 / 24$ & $29 / 59$ & $18 / 24$ & $4 / 11$ & $62 / 29$ & $28 / 59$ & $13 / 50$ \\
\hline & 2006 & $39 / 36$ & $0 / 11$ & $92 / 100$ & $100 / 10$ & $97 / 100$ & $10 / 45$ & - & $10 / 17$ & $18 / 28$ & $2 / 20$ & $6 / 5$ & $46 / 40$ & $18 / 45$ & $5 / 25$ \\
\hline & 2007 & $60 / 42$ & $0 / 3$ & $87 / 90$ & $97 / 94$ & $96 / 100$ & $25 / 49$ & - & $4 / 12$ & $7 / 9$ & $8 / 9$ & $12 / 21$ & $51 / 36$ & $30 / 48$ & $7 / 27$ \\
\hline & & $146 / 120$ & $0 / 4$ & $93 / 96$ & $99 / 98$ & $97 / 99$ & $20 / 46$ & - & $9 / 18$ & $14 / 32$ & $10 / 18$ & $8 / 13$ & $53 / 35$ & $26 / 51$ & $8 / 34$ \\
\hline \multirow[t]{2}{*}{ Citrobacter spp. } & 2005 & $9 / 7$ & $0 / 14$ & $91 / 100$ & $91 / 100$ & $100 / 10$ & $0 / 71$ & - & $0 / 14$ & $22 / 43$ & $0 / 43$ & $0 / 0$ & $0 / 0$ & $11 / 71$ & $0 / 29$ \\
\hline & 2006 & $20 / 21$ & $5 / 11$ & $90 / 85$ & $95 / 90$ & $100 / 10$ & $40 / 50$ & - & $25 / 15$ & $35 / 15$ & $20 / 20$ & $0 / 24$ & $5 / 16$ & $40 / 65$ & $25 / 20$ \\
\hline
\end{tabular}

$\mathrm{A}=$ Amikacin; $\mathrm{AUG}=$ amoxicillin/clavulanic acid; $\mathrm{AMP}=$ ampicillin $; \mathrm{CF}=$ cepholathin $\mathrm{CTX}=$ cefotaxime; $\mathrm{CXM}=$ cefuroxime; $\mathrm{CIP}=$ ciprofloxacin; SXT = cotrimoxazole; GN = gentamicin; NA = nalidixic acid; NIT = nitrofurantoin; PIP = piperacillin; TZ = piperacillin/tazobactam.

\section{Discussion}

Analysis of our results showed that $26.6 \%$ of the urine samples from inpatients and outpatients yielded significant bacteriuria. A majority of the UTIs in our study occurred in adult patients (51\%), mainly women (73\%), thereby confirming the previous report that adult women have a higher prevalence of UTI than men, principally owing to anatomic and physical factors [1]. The prevalence encountered in the present study was $26.6 \%$; a finding considerably higher than other reports of studies $[6$, 7] emanating from Kuwait which demonstrated the prevalence of significant bacteriuria as $9.7 \%$ [6] and $11.4 \%$ [7]. The explanation for this is partly due to the fact that the criterion used for determining significant bacteriuria was lower $\left(\geq 10^{4} \mathrm{CFU} / \mathrm{ml}\right)$ in the current study than that used in other studies $\left(\geq 10^{5} \mathrm{CFU} / \mathrm{ml}\right)$. In addition, growth of 2 bacteria was also categorized as significant if both were present at $\geq 10^{4} \mathrm{CFU} / \mathrm{ml}$ of urine. In this study, the etiological agents of UTI essentially correlate well with a previous study from Kuwait [6].

Our data indicate that $E$. coli is still the most frequent uropathogen causing UTI in the community and hospital settings. Thus, our finding is consistent with those of previous studies in which E. coli was the predominant pathogen isolated from both CA-UTI and HA-UTI [6, 7]. Also our study showed that Candida spp. were the second most common isolates from patients with nosocomial UTI, a finding in concordance with data from other studies that have reported an increase in the number of Candida spp. 
Table 4. Frequency and percentage of resistant Gram-positive uropathogens isolated from outpatient vs. inpatient UTI cases

\begin{tabular}{|c|c|c|c|c|c|c|c|c|c|c|c|}
\hline \multirow[t]{2}{*}{ Bacteria } & \multirow[t]{2}{*}{ Year } & \multirow{2}{*}{$\begin{array}{l}\text { Total No. of } \\
\text { isolates CA/HA }\end{array}$} & \multicolumn{9}{|c|}{ Percentage of resistant uropathogens of CA-UTI vs. HA-UTI } \\
\hline & & & AMP & $\mathrm{CF}$ & $\mathrm{CC}$ & Clox & SXT & $\mathrm{P}$ & Van & $\mathrm{E}$ & NIT \\
\hline \multirow{3}{*}{ S. agalactiae } & 2006 & $351 / 93$ & $0 / 0$ & - & $0 / 0$ & - & - & $0 / 0$ & $0 / 0$ & $21 / 18$ & $0 / 0$ \\
\hline & 2007 & $366 / 108$ & $0 / 0$ & - & $0 / 0$ & - & - & $0 / 0$ & $0 / 0$ & $17 / 17$ & $0 / 0$ \\
\hline & & & $0 / 0$ & - & $0 / 0$ & - & - & $0 / 0$ & $0 / 0$ & $16 / 15$ & $0 / 0$ \\
\hline \multirow{3}{*}{ Enterococcus spp. } & 2006 & $302 / 237$ & $2 / 1$ & - & - & - & - & $32 / 39$ & $0 / 0$ & - & $2 / 3$ \\
\hline & 2007 & $185 / 245$ & $1 / 4$ & - & - & - & - & $30 / 55$ & $0 / 0$ & - & $2 / 4$ \\
\hline & & & $1 / 3$ & - & - & - & - & $24 / 35$ & $0 / 0$ & - & $2 / 3$ \\
\hline \multirow[t]{2}{*}{ S. aureus } & 2005 & $41 / 14$ & - & $0 / 0$ & $0 / 0$ & $0 / 0$ & $2 / 4$ & $78 / 93$ & $0 / 0$ & $2 / 14$ & $0 / 0$ \\
\hline & 2006 & $38 / 32$ & - & $0 / 0$ & $9 / 14$ & $0 / 0$ & $3 / 0$ & $91 / 90$ & $0 / 0$ & $11 / 17$ & $0 / 0$ \\
\hline \multirow{2}{*}{ S. epidermidis } & 2007 & $10 / 5$ & - & $83 / 67$ & $50 / 33$ & $83 / 67$ & $20 / 40$ & $83 / 90$ & $0 / 0$ & $67 /-$ & $0 / 0$ \\
\hline & & & - & $61 / 69$ & $45 / 25$ & $61 / 69$ & $10 / 51$ & $78 / 88$ & $0 / 0$ & $54 / 46$ & $0 / 0$ \\
\hline \multirow[t]{4}{*}{ S. saprophyticus } & 2005 & $9 / 1$ & - & $0 / 0$ & $0 / 0$ & $0 / 0$ & $0 / 0$ & $78 / 0$ & $0 / 0$ & $62 / 0$ & $0 / 0$ \\
\hline & 2006 & $13 /-$ & - & $0 /-$ & $0 /-$ & $0 /-$ & $0 /-$ & $83 /-$ & $0 /-$ & $33 /-$ & $0 /-$ \\
\hline & 2007 & $9 / 2$ & - & $33 / 50$ & $11 / 50$ & $33 / 50$ & $0 / 0$ & $78 / 100$ & $0 / 0$ & $44 / 50$ & $0 / 0$ \\
\hline & & & - & $10 / 33$ & $3 / 33$ & $10 / 33$ & $0 / 0$ & $80 / 67$ & $0 / 0$ & $45 / 33$ & $0 / 0$ \\
\hline
\end{tabular}

$\mathrm{AMP}=$ Ampicillin; $\mathrm{CF}=$ cephalothin; $\mathrm{CC}=$ clindamycin; Clox = cloxacillin; $\mathrm{SXT}=$ cotrimoxazole $\mathrm{P}=$ penicillin; Van = vancomycin; $\mathrm{E}=$ erythromycin; NIT = nitrofurantoin; - = not tested.

Table 5. Percentage of susceptible isolates of ESBL-producing E. coli and ESBL-producing K. pneumoniae to antimicrobials (inpatients/ outpatients)

\begin{tabular}{|c|c|c|c|c|c|c|}
\hline & 2005 & & 2006 & & 2007 & \\
\hline & ESBL-pos & olates, \% & ESBL-po & solates, $\%$ & ESBL-po & isolates, \% \\
\hline & E. coli & K. pneumoniae & E. coli & K. pneumoniae & E. coli & K. pneumoniae \\
\hline Amc & $12 / 41$ & $35 / 38$ & $33 / 47$ & $40 / 47$ & $60 / 10$ & $9 / 39$ \\
\hline NA & $26 / 30$ & $80 / 94$ & $23 / 40$ & $73 / 83$ & $16 / 21$ & $14 / 6$ \\
\hline A & $97 / 100$ & $100 / 100$ & $96 / 99$ & $79 / 70$ & $96 / 96$ & $94 / 100$ \\
\hline GN & $40 / 47$ & $20 / 28$ & $54 / 54$ & $37 / 50$ & $54 / 40$ & $58 / 37$ \\
\hline NIT & $84 / 88$ & $45 / 31$ & $90 / 88$ & $7 / 13$ & $89 / 81$ & $22 / 9$ \\
\hline CIP & $4 / 12$ & $40 / 19$ & $32 / 3$ & $10 / 20$ & $29 / 24$ & $9 / 3$ \\
\hline PIP & $1 / 3$ & $0 / 6$ & $3 / 3$ & $20 / 3$ & $1 / 1$ & $0 / 3$ \\
\hline $\mathrm{TZ}$ & $91 / 94$ & $85 / 100$ & $95 / 100$ & $85 / 93$ & $85 / 97$ & $72 / 91$ \\
\hline SXT & $53 / 30$ & $50 / 31$ & $33 / 18$ & $69 / 60$ & $37 / 43$ & $47 / 74$ \\
\hline Fox & $80 / 100$ & $95 / 100$ & $82 / 78$ & $73 / 90$ & $83 / 78$ & $72 / 97$ \\
\hline Mem & $100 / 100$ & $100 / 100$ & $100 / 100$ & $100 / 100$ & $100 / 100$ & $100 / 100$ \\
\hline
\end{tabular}

Amc = Amoxicillin-clavulanic acid; NA = nalidixic acid; $\mathrm{A}=$ amikacin; $\mathrm{GN}=$ gentamicin; $\mathrm{NIT}=$ nitrofurantoin; $\mathrm{CIP}=$ ciprofloxacin; PIP = piperacillin; TZ = piperacillin/tazobactam; SXT = cotrimoxazole; Fox = cefoxitin; Mem = meropenem. 
in nosocomial UTI $[6,8]$. The high prevalence of yeast as a cause of UTI may be related to the type of patients, advances in surgical and medical management of the patients as well as excessive use of broad-spectrum antibiotics, high prevalence of diabetes mellitus and obesity in the Kuwait population [9]. This is supported by the findings in the 2001 ESCMID Study Group report on a European perspective study on nosocomial UTI, where Candida spp. were the third most commonly isolated pathogen [8]. It is noteworthy that the high prevalence rate of S. agalactiae in CA-UTI and HA-UTI at Al-Amiri Hospital is consistent with the findings of previous studies in Kuwait which also showed high prevalence rates of $S$. agalactiae $[6,7]$. However, there is scarcely any other report that has published such a high prevalence rate of $S$. agalactiae in UTI as observed in our study. For example, the ESCMID Study Group report demonstrated only a 1.6\% prevalence rate of $S$. agalactiae in UTI among patients in the non-EU countries [8]. The explanation for this observation may be related to the high prevalence of diabetes mellitus among the Kuwaiti population [9]. In a previous study, $36 \%$ of the patients with UTI had diabetes mellitus [10] and this disease is a known predisposing factor for S. agalactiae infection in adults. In addition, previous experience shows that there is a high vaginal colonization rate of pregnant and nonpregnant individuals by $S$. agalactiae in Kuwait [11]. Enterococci were the fourth most common isolate causing UTI in hospitalized patients, which is slightly different from the findings in previous studies in Kuwait that showed that enterococci ranked fifth among the UTI pathogens. There was a low prevalence of S. saprophyticus $(0.3 \%)$, which is less than the previous reports of $2 \%$ each from Kuwait [6] and the UK [2]. However, recent reports from the USA have found this pathogen to be present in $4.0-9.7 \%$ of the women with uncomplicated cystitis [12]. This finding may indicate a distinct pattern of Gram-positive uropathogen distribution in our area. The finding of uropathogens resistant to many commonly used antibiotics is a new experience in this hospital. In general, the resistance rates of Gram-negative bacteria were relatively lower in outpatients than those of inpatients, a finding which is consistent with observations all over the world $[11,13]$. The differences in antibiotic susceptibility between isolates from community and hospital patients may be attributable to the presence of additional risk factors for antibiotic resistance in the latter group. These include frequent antibiotic use, underlying pathology and presence of invasive devices. The high resistance rates of $E$. coli and other members of the family Enterobacteriaceae to several

In vitro Activity of Several Antibiotics against Uropathogens in Amiri Hospital commonly used antibiotics are alarming. For example, the resistance of $E$. coli from CA-UTI and HA-UTI to commonly used oral antibiotics is at an unacceptably high level. The fluoroquinolone resistance in $E$. coli was much higher than those reported in several recent studies in Kuwait [6], Europe and Canada [14]. This high resistance level might have been driven by prior exposure to fluoroquinolones which has been recently acknowledged as an independent risk factor for ciprofloxacin-resistant E. coli from CA-UTI [15]. Ciprofloxacin is one of the most prescribed antibiotics in Kuwait particularly by the general practitioners. This study shows that there is a relatively high prevalence of $E$. coli and K. pneumoniae in Al-Amiri Hospital which is higher than that reported from other countries in this region $[16,17]$.

Our data showed a substantial reduction in susceptibility to antibiotics frequently used in the community. Ampicillin, amoxicillin-clavulanic acid, cephalothin, TMPSMX and ciprofloxacin did not have good in vitro coverage for many of the uropathogens isolated in this study. Because of this, they are of little use as empirical therapeutic options in outpatient departmental setting. Nitrofurantoin is the only oral agent that remains relatively active against most uropathogens and can be used for empiric therapy in uncomplicated UTI, particularly in the community. However, nitrofurantoin is not traditionally used in UTI with any significant tissue involvement such as pyelonephritis or infections caused by K. pneumoniae or Proteus spp. The current data may reflect the increased use of orally prescribed agents in our region, to treat hospital- or community-acquired infections, such as UTI or respiratory tract infections. This overuse may select for multidrug-resistant $E$. coli phenotypes, harboring the potential to disseminate within our region. Further antimicrobial consumption studies at the community level are needed in our region to verify this assumption. In general, carbapenems, piperacillin-tazobactam and amikacin agents showed excellent in vitro coverage of the uropathogens isolated in this study. This information will be useful in choosing empiric therapy for seriously ill hospitalized patients with suspected urosepsis.

We are aware that many CA-UTIs are treated without bacteriological testing, and most isolates from the community that are tested in our laboratory may be predominantly from patients for whom previous antimicrobial treatment failed or from patients with other underlying risk factors. Therefore, the results of our study may not represent the actual distribution and antibiotic resistance pattern of the uropathogens causing acute uncomplicated CAUTIs and may not necessarily serve as a basis to develop 
guidelines for the empirical treatment of CA-UTIs. However, they emphasize the need to investigate the distribution and susceptibility pattern of pathogens causing uncomplicated CA-UTI which would expectedly indicate the most appropriate antibiotic for empirical treatment of this condition. In addition, the results of our study increased our knowledge about the types of pathogen responsible for UTI in hospitalized patients with CA-UTI and HA-UTI and their resistance patterns to antibiotic drugs. This knowledge would help clinicians choose the right empirical treatment, particularly in the hospital setting.

The high prevalence of ESBL-producing E. coli and $K$. pneumoniae causing UTI in our hospital has reached an alarming proportion. There was an increasing trend in ESBL-producing $E$. coli isolates from outpatients from $9 \%$ in 2005 to $17 \%$ in 2007 . The prevalence of the inpatient isolates decreased from $30 \%$ in 2005 to $27 \%$ in 2007. However, the trend in K. pneumoniae resistance increased from 12\% in CA-UTI in 2005 to $19 \%$ in 2007 and from $17 \%$ in HA-UTI in 2005 to $33 \%$ in 2007 . A compounding finding is the fact that the majority of our ESBL-producing E. coli and $K$. pneumoniae strains were multiresistant. Specifically, most were resistant to the quinolones, amoxicillin-clavulanic acid and TMP-SMX. This associated resistance to other classes of antimicrobials is especially problematic in urinary isolates and underscores the therapeutic challenge that they represent.

\section{Conclusion}

This study demonstrated that almost a third of all the Gram-negative isolates reported in this study were resistant to $\geq 4$ antibiotics, but the carbapenems, piperacillintazobactam and amikacin demonstrated excellent in vitro coverage for the uropathogens. Nitrofurantoin is the only oral agent that remains relatively active against most uropathogens and can be used for empiric therapy in uncomplicated UTI, particularly in the community setting. In the present study a large number of E. coli and K. pneumoniae was ESBL producers and multidrug resistant. Monitoring of ESBL production and antimicrobial susceptibility testing are necessary to avoid treatment failure in patients with UTI. To optimize the use of empirical therapy, evidence-based guidelines for antibiotics use, guided by surveillance studies of target bacteria, need to be developed and implemented.

\section{References}

1 Sussman M: Urinary tract infections; in Collier L, Balows A, Sussman M (eds): Topley \& Wilson's Microbiology and Microbial Infections, ed 9. London, Arnold, 1998, pp 601621.

-2 Foxman B: Epidemiology of urinary tract infections: incidence, morbidity, and economic costs. Am J Med 2002;113(suppl 1A):5-13.

$\checkmark 3$ Manges AR, Natarajan P, Solberg OD, Dietrich PS, Riley LW: The changing prevalence of drug-resistant Escherichia coli clonal groups in a community: evidence for community outbreaks of urinary tract infections. Epidemiol Infect 2006;134:425-431.

4 Clinical and Laboratory Standards Institute: Performance standards for antimicrobial susceptibility testing, 15th informational supplement (M100-S15). Wayne, CLSI, 2005.

5 Al Sweih N, Jamal W, Rotimi VO: Spectrum and antibiotic resistance of uropathogens isolated from hospital and community patients with urinary tract infections in two large hospitals in Kuwait. Med Princ Pract 2005; 14:401-407.

$\checkmark 6$ Dimitrov TS, Udo EE, Emara M, Awini F, Passadilla R: Etiology and antibiotic susceptibility patterns of community-acquired urinary tract infections in Kuwait hospital. Med Princ Pract 2003;13:334-339.
7 ESCMID Study Group Report: A European perspective on nosocomial urinary tract infections. I. Report on the microbiology workload, etiology and antimicrobial susceptibility (ESGNI-003 study). Clin Microbiol Infect 2001;7:523-531.

8 Moussa MA, Alsaeid M, Abdella N, Refai TM, Al-Sheikh N, Gomez JE: Prevalence of type 2 diabetes mellitus among Kuwaiti children and adolescents. Med Princ Pract 2008; 17:270-275

$\checkmark 9$ Dobardzic AM, Dobardizic R: Epidemiological features of complicated UTI in a district hospital of Kuwait. Eur J Epidemiol 1997;13. 465-470.

10 Al-Sweih N, Hammoud M, Al-Shimmiri M, Jamal M, Neil L, Rotimi V: Serotype distribution and mother-to-baby transmission rate of Streptococcus agalactiae among expectant mothers in Kuwait. Arch Gynecol Obstet 2005;272:131-135.

11 Gupta K, Scholes D, Stamm WE: Increasing prevalence of antimicrobial resistance among uropathogens causing acute uncomplicated cystitis in women. JAMA 1999;281:736-738.

12 Kahlmeter G: An international survey of the antimicrobial susceptibility of pathogens from uncomplicated urinary tract infections: the ECOSENS Project. I Antimicrob Chemother 2003;51:69-76.
13 Dornbusch K, King A, Legakis N, European Study Group on Antibiotic Resistance (ESGAR): Incidence of antibiotic resistance in blood and urine isolates from hospitalized patients: report from a European collaborative study. Scand J Infect Dis 1998;30:281-288.

14 Killgore KM, March KL, Guglielmo BJ: Risk factors for community-acquired ciprofloxacin-resistant Escherichia coli urinary tract infection. Ann Pharmacother 2004;38:11481152

15 Kader AA, Kumar A: Prevalence and antimicrobial susceptibility of extended-spectrum $\beta$-lactamase-producing Escherichia coli and Klebsiella pneumoniae in a general hospital. Ann Saudi Med 2005;25:239-242.

16 Mansouri S, Shareifi S: Antimicrobial resistance pattern of Escherichia coli causing urinary tract infections, and that of human fecal flora, in the southeast of Iran. Microb Drug Resist 2002;8:123-128.

17 Kehinde EO, Rotimi VO, Al-Hunayan A, Abdul-Halim H, Boland F, Al-Awadi K: Bacteriology of urinary tract infection associated with indwelling ureteral J stent. J Endourol 2004; 18:891-896. 